\title{
Interferência contextual e nível de habilidade na aprendizagem do serviço do voleibol
}

CDD. 20.ed. 152.3

796.325
Domingos Manuel NHAMUSSUA*

António PRISTA*

Luciano BASSO**

Go TANI**

\section{Resumo}

Este estudo investigou o efeito da interferência contextual (IC) na aquisição do serviço do voleibol em adolescentes com diferentes niveis de habilidade, considerando os testes de transferência imediato e retardado. A amostra foi composta por 56 meninas com idade entre 14 e 16 anos, distribuídas em quatro grupos de prática: aleatório com nivel superior de habilidade (GAS), blocos com nível superior de habilidade (GBS), aleatório com nível inferior de habilidade (GAI) e blocos com nivel inferior de habilidade (GBI). A tarefa motora consistiu dos serviços por baixo e por cima direcionados a alvos na quadra. Os resultados mostraram que o efeito da IC não foi observado nos grupos GAI e GBI, por outro lado, os grupos GAS e GBS apresentaram melhor desempenho no teste de transferência imediato. A ausência de diferenças significativas no teste de transferência retardado sugere que o efeito da IC pode ser temporário.

UnITERMOS: Prática variada; Aprendizagem motora; Nivel de habilidade.

\section{Introdução}

Sabendo-se que o uso de métodos baseados apenas na tradição não mais garante o sucesso na formação e treinamento desportivos, a aplicação de conhecimentos académico-científicos na intervenção constitui um dos pressupostos de sucesso nas profissões academicamente orientadas (TANI, 2002, 2008). Nesta óptica, nas últimas décadas, a organização dos contextos de práctica na aquisição de habilidades motoras tem merecido uma atenção especial, procurando-se uma ligação às teorias da Aprendizagem Motora.

Os estudos realizados nesta temática têm direcionado o seu enfoque na variabilidade da prática, tendo em conta a natureza e as características da tarefa em estudo. Eles têm sido realizados com base em duas abordagens teóricas: a teoria de esquema (SCHMIDT, 1975) e o princípio de interferência contextual (BATTIG, 1972). Em ambos os casos, a práctica de habilidades em condições variáveis é vista como mais eficaz do que em condiçōes de prática constante (TEMPRADO, 1997).

O efeito da interferência contextual (EIC) refere-se ao grau em que a práctica de várias habilidades interfere na aprendizagem das mesmas, e ocorre quando algumas variaçóes de uma tarefa devem ser aprendidas durante a prática com altos níveis de interferência (prática variada aleatória). A interferência contextual pode ser elevada ou baixa. A elevada interferência contextual ocorre quando as tarefas a serem aprendidas, por exemplo, $\mathrm{A}, \mathrm{B}$ e $\mathrm{C}$, são pracicadas de forma aleatória (A,C,A,B,B,A,B,C,A) e baixa quando as mesmas são praticadas em blocos (A,A,A; B,B,B; C,C,C).

Shea e Morgan (1979) foram os primeiros a investigar o EIC na aprendizagem de habilidades motoras e obtiveram confirmação dos resultados encontrados por BATTIG (1972) na aprendizagem verbal. A partir de então, várias pesquisas têm sido realizadas com habilidades motoras, mas não existe ainda consenso sobre a superioridade desta prática quando se considera a idade e o nível de habilidade dos sujeitos, as características da tarefa e a quantidade de interferência (BRADY, 1998). Além disso, não é muito clara a transferência dos resultados obtidos nas condiçôes laboratoriais para situações do mundo real (BRAdy, 1997; MEIRA JúNIOR \& TANI, 2001). O foco do presente estudo é a investigação do EIC em
*Faculdade de Educação Física e Desporto, Universidade Pedagógica de Maputo - Moçambique.

**Escola de Educação Física e Esporte,

Universidade de São

Paulo.

A


relação ao nível de habilidade, visto que a literatura ainda não mostra resultados conclusivos (JONES \& French, 2007; Pollock \& LeE, 1997). Nos estudos de Hall, Domingues e Cavazos (1994) com beisebol e Guadagnoli, Holcomb e Weber (1999) com golfe, ambos feitos com atletas de alto nível, o EIC foi verificado. No entanto, nos estudos de GoOdwIN e MEEuwsen (1996) com golfe e Herbert, LANDin e Solmon (1996) com tênis, ambos com sujeitos adultos, mas não atletas, o EIC não foi verificado. O estudo de Landin e Herbert (1997), por sua vez, mostrou que a combinação das duas práticas é melhor para sujeitos de habilidade moderada. Isto é, não há consistência de resultados do EIC quando se considera o nível de habilidade dos sujeitos. Além disso, estudos realizados com a mesma modalidade

\section{Método}

\section{Participantes}

A amostra foi constituída por 56 alunas do Ensino Secundário nas escolas da Cidade de Maputo, na faixa etária entre 14 e 16 anos. Essa faixa etária foi escolhida para tornar possível a formação dos dois grupos de prática diferenciados por níveis de habilidade, ou seja, com nível superior e inferior de habilidade, o que pressupunha participantes já iniciadas nessa modalidade desportiva. Elas constituíram quatro grupos experimentais em função do tipo de prática e nível de habilidade, nomeadamente: grupo de prática aleatória $(\mathrm{n}=12) \mathrm{com}$ nível superior de habilidade (GAS); grupo de prática em blocos $(\mathrm{n}=14)$ com nível superior de habilidade (GBS); grupo de prática aleatória $(\mathrm{n}=16)$ com nível inferior de habilidade (GAI); e grupo de prática em blocos ( $\mathrm{n}=14$ ) com nível inferior de habilidade (GBI). Para a seleção das alunas foi realizado um pré-teste que consistiu na execução de cinco serviços por baixo a 5 $\mathrm{m}$ da rede ou da linha de fundo, para o alvo 2 descrito a seguir. Os pontos obtidos por cada sujeito nas cinco tentativas foram somados para efeitos de classificação de todos em ordem crescente ("ranking"). Participaram no pré-teste 100 alunas, das quais 48 foram selecionadas, sendo 24 com o nível inferior de habilidade e $24 \mathrm{com}$ o nível superior. Para garantir a formação de grupos com níveis diferentes de habilidade, 52 alunas com resultados intermediários foram excluídas. Consoante o nível de habilidade, os 24 sujeitos foram alocados nos dois grupos de prática de modo a formar grupos homogêneos. Desta forma, nos 24 do nível inferior de do presente estudo (voleibol) em populações de características semelhantes no tocante a estágio de desenvolvimento, não confirmaram (FRENCH, RINK \& Werner, 1990; Meira Júnior, 1999; UgrinoWitsCh, 1997; Zetou, Michalopoulou, GiaZiTZI \&Kioumourtzoglou, 2007) ou confirmaram apenas parcialmente o EIC (TRAVlos, 2010). Estudo com a mesma habilidade, mas com população de idade um pouco mais avançada (ensino médio) também não confirmou o EIC (JONES \& FrENCH, 2007). Assim, o objetivo deste estudo é investigar os efeitos de dois tipos de prática variada (aleatória e em blocos) na aquisição da habilidade de serviço no voleibol, numa situação de campo, comparandose dois grupos de escolares formados por níveis de habilidade - alto e baixo.

habilidade, o $1^{\circ}$ e o $24^{\circ}$ foram alocados para o grupo aleatório, o $2^{\circ}$ e o $23^{\circ}$ para grupo em blocos, o $3^{\circ} \mathrm{e}$ o $22^{\circ}$ para o grupo aleatório e assim sucessivamente. Todas as participantes foram informadas sobre os objetivos da pesquisa pelo responsável do estudo e tiveram a autorização dos encarregados de educação através da assinatura de um formulário de consentimento. $\mathrm{O}$ projeto, no que se refere a aspectos éticos, foi aprovado pelo Conselho Científico da Faculdade de Educação Física e Desporto da Universidade Pedagógica, Moçambique.

\section{Tarefa motora}

A tarefa consistiu em realizar o serviço do voleibol a partir de um lado do campo, fazendo-o passar por cima da rede (FIGURA 1) em direção a um alvo, com o objetivo de alcançar a maior pontuação possível, numa situação adaptada do teste de precisão da AAHPER (1969). Os alvos foram colocados num plano horizontal, desenhados sobre um tecido de 4 x 4 metros, com zonas de pontuação circulares demarcadas por tinta verde. A largura das linhas seguiu o padrão do campo de voleibol, ou seja, $5 \mathrm{~cm}$.

Para efeitos de pontuação, o centro de cada alvo implicava a pontuação 10 e média um metro de diâmetro; as outras três áreas tiveram um metro a mais de diâmetro em relação à área central, com valores de pontuação de 8,6 e 4 pontos, respectivamente. Como medida complementar, foi criada uma área de pontuação secundária que valia 
2 pontos, isso para privilegiar os serviços executados na direcção do alvo, mas sem acertá-lo. A área foi delimitada por duas linhas originárias com ponto central no local de serviço, cada qual tangenciando o limite externo do alvo circular (em forma de "V") até um limite de dois metros após a linha de fundo.

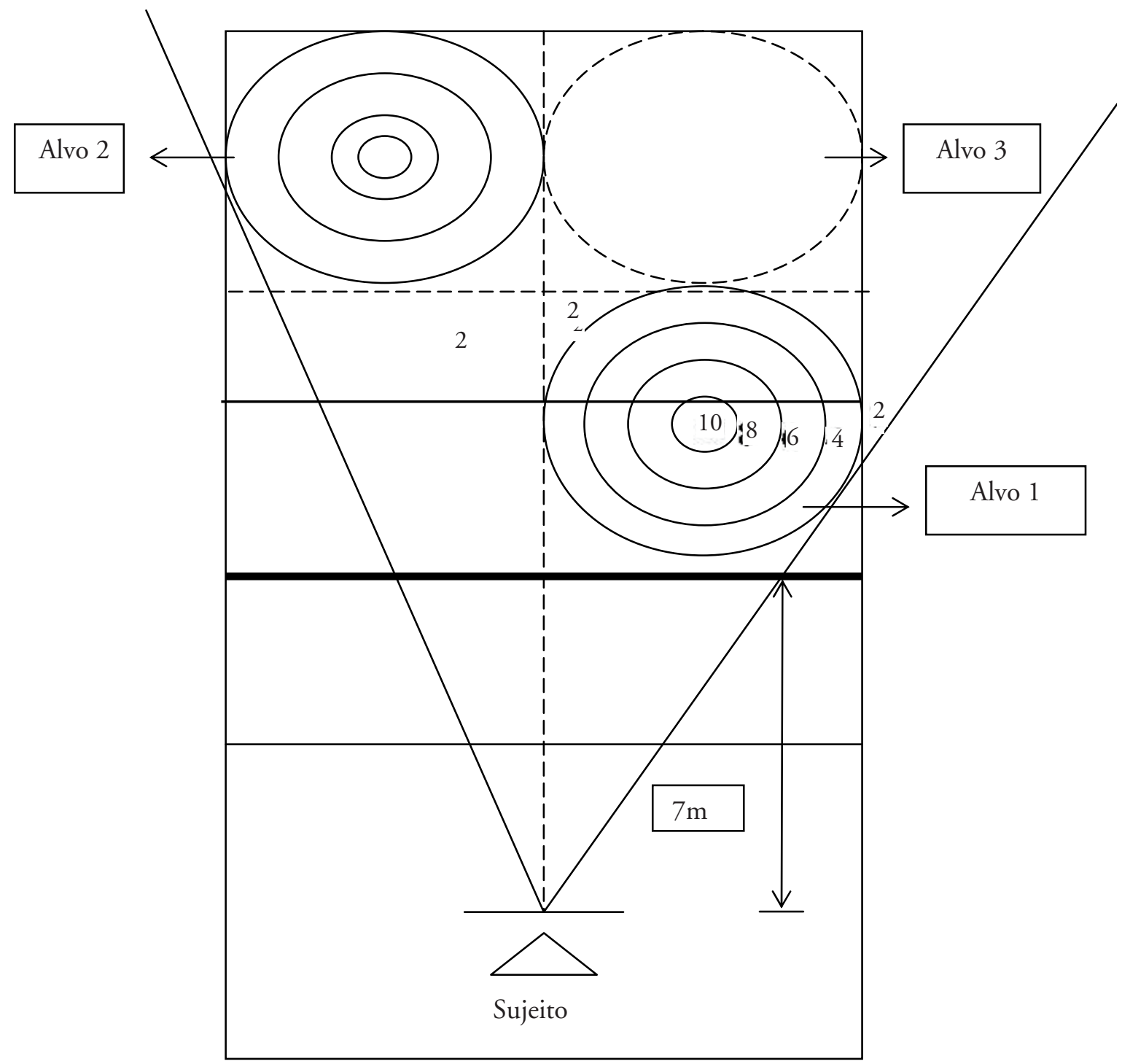

FIGURA 1 - Teste de precisão do serviço no voleibol (Adaptado da AAHPER, 1969).

Durante a aquisição foram utilizados dois tipos de serviços (por baixo e por cima, ou seja, tipo tênis) (FIGURA 2a e 2b) para dois alvos (1 e 2), enquanto que nos testes de transferência foi utilizado o serviço por cima direcionado para um novo alvo (3). Durante a pesquisa cada serviço foi considerado como uma tentativa, independentemente do resultado, sendo que as tentativas em que a bola caía na rede, tocava nas antenas laterais, ou caia fora da zona delimitada (antes ou depois da rede) foram consideradas como erradas, com a atribuição de zero ponto. $\mathrm{O}$ intervalo intertentativas foi de aproximadamente cinco segundos. Participantes de todos os grupos receberam conhecimento de resultados acerca da pontuação obtida em todas as tentativas da fase de aquisição. Nos testes de transferência o mesmo não foi fornecido.

\section{Delineamento experimental}

A pesquisa foi dividida em quatro etapas: préteste, fase de aquisição, teste de transferência imediato e retardado. O pré-teste consistiu na realização de cinco serviços por baixo a $5 \mathrm{~m}$ da rede para o alvo 2 conforme a FIGURA 1, em uma sessão apenas. A fase de aquisição consistiu na execução de 24 serviços em oito sessões, sendo três por semana, totalizando 192 tentativas no experimento. $\mathrm{O}$ teste de transferência imediato foi realizado no final da 
última sessão da fase de aquisição ( $8^{a}$ sessão) com oito tentativas. $\mathrm{O}$ teste de transferência retardado foi realizado 72 horas após a fase de aquisição, tendo sido a mesma tarefa do teste de transferência imediato.

Um estudo piloto foi realizado com o objetivo de testar a adequação dos tipos de serviço, posição dos alvos no campo, local do serviço, número de tentativas, gestão de tempo entre as tentativas e registro dos resultados.

\section{Variáveis e tratamento estatístico}

O presente estudo teve como variáveis independentes o tipo de prática (aleatória e em blocos) e o nível de habilidade (superior e inferior), e como variável dependente a precisão da execução (pontuação) nas diferentes sessões de prática. Para a análise dos resultados foi usado o pacote estatístico SPSS versão 11.5. A estatística inferencial constou da aplicação de uma ANOVA para medidas repetidas (oito blocos, correspondentes a oito sessões de prática) em cada grupo, para identificar as diferenças entre os blocos da fase de aquisição. Já para o teste de transferência foi aplicada uma ANOVA mista "two-way" (dois tipos de prática $\mathrm{x}$ três blocos) para cada nível de habilidade, sendo analisados o último bloco da fase de aquisição e os dois dos testes de transferência. Em todas as analises utilizou-se do $\mathrm{p}<0,05$. Para encontrar o local das diferenças foram utilizados o teste "Post Hoc" de Tukey HSD (efeitos principais) e de Bonferroni (efeito de interação).

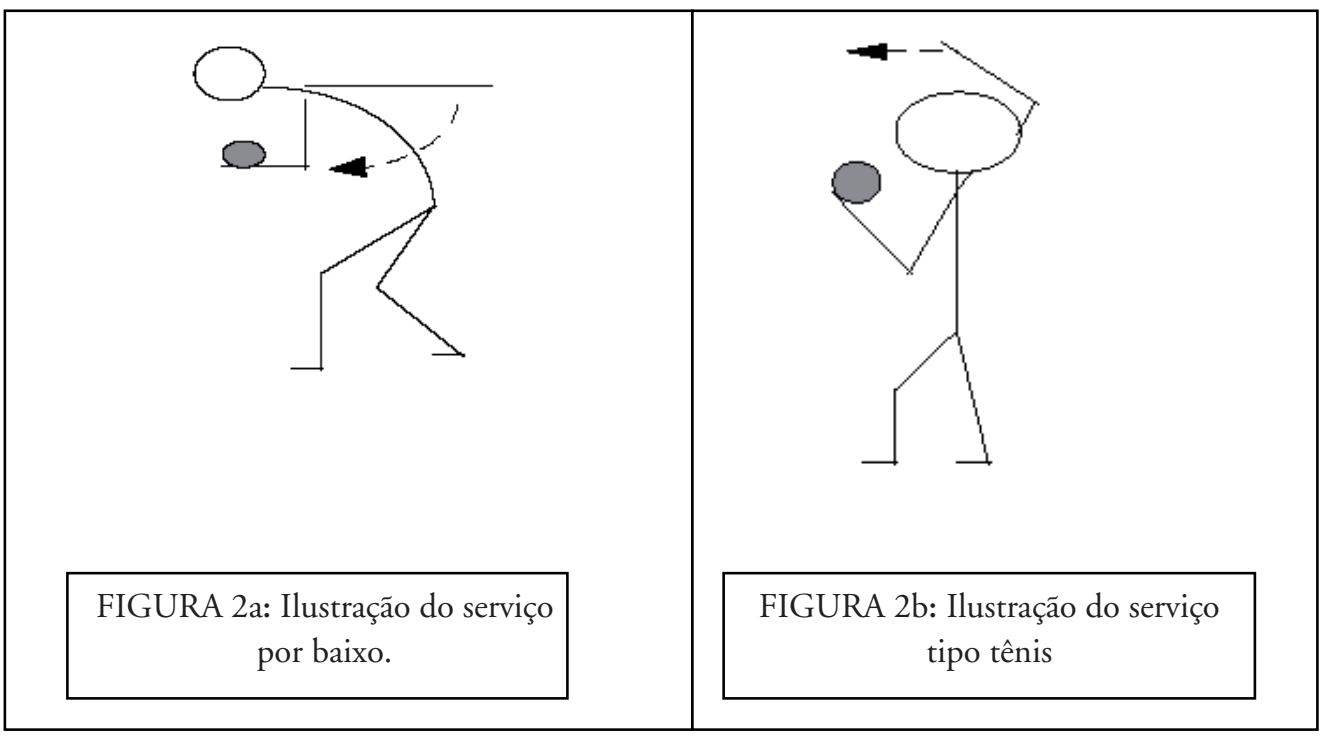

FIGURA 2 -Tarefas motoras utilizadas.

\section{Resultados}

A FIGURA 3 apresenta as curvas de desempenho dos quatro grupos na fase de aquisição e testes de transferência. $\mathrm{Na}$ primeira observação pode-se constatar que as curvas dos dois tipos de prática (aleatório e em blocos) em cada nível de habilidade (inferior e superior) são semelhantes na fase de aquisição, isto é, em sujeitos com mesmo nível de habilidade foram observados níveis de desempenho similares, tanto no grupo de prática aleatória como em blocos. 


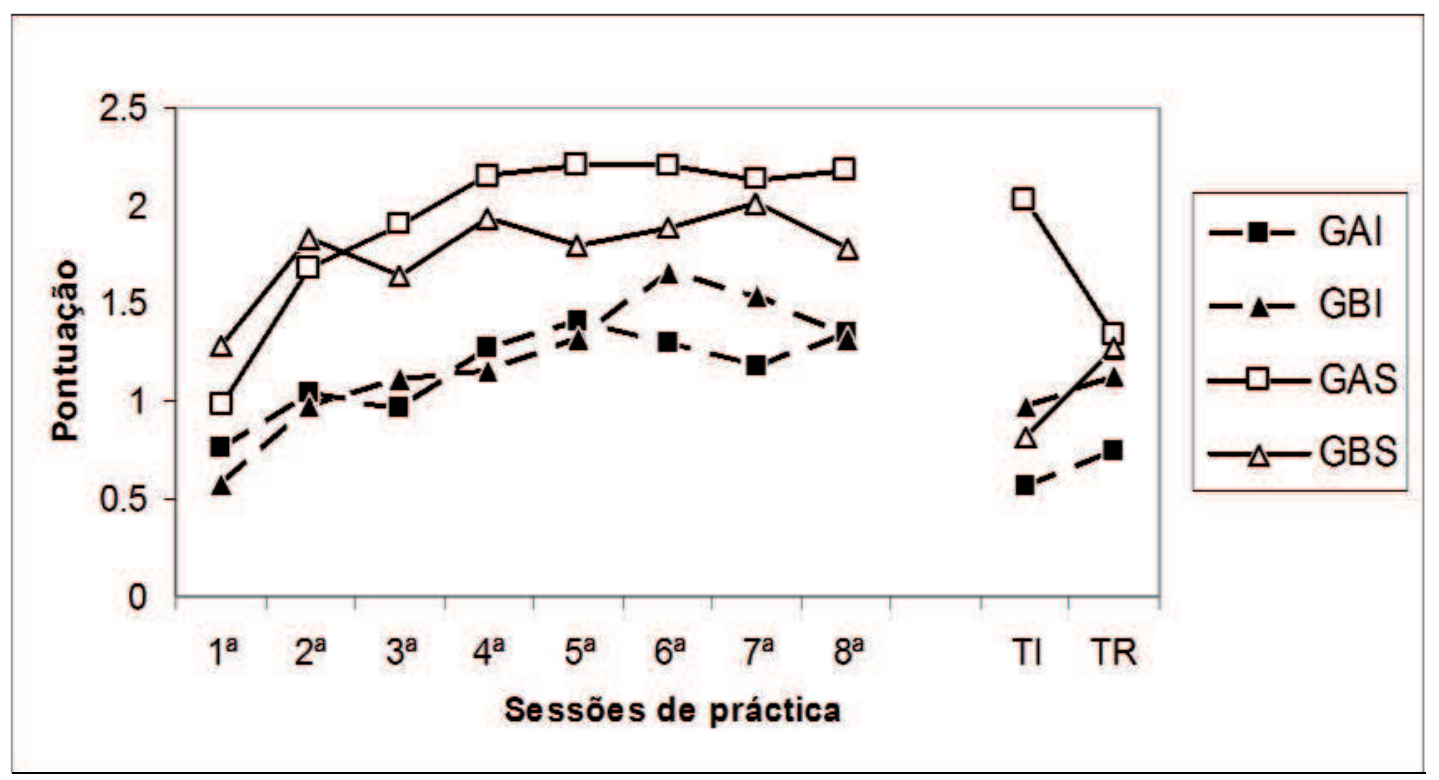

FIGURA 3 -Média de pontos ao longo das sessões de prática (fase de aquisição e testes de transferência) para os grupos de habilidade inferior com prática aleatória (GAI) e em blocos (GBI), e com habilidade superior com prática aleatória (GAS) e em blocos (GBS).

\section{Fase de aquisição}

Para efeitos de inferência dos resultados na fase de aquisição, checou-se a normalidade da distribuição dos dados por meio do teste Shapiro-Wilk, o qual não indicou violação desse pressuposto em nenhum dos blocos. No GAI foram encontradas diferenças significativas entre as oito sessōes $(F[7 ; 105]=3,20 ; p=0,004)$. Contudo, o teste "Post Hoc" não detectou as sessōes que produzem essas possíveis diferenças. No GBI foram encontradas igualmente diferenças significativas entre as sessões ( $\mathrm{F}$ $[7 ; 91]=10,0 ; p=0,0001)$. O teste "Post Hoc" mostrou que a $1^{\text {a }}$ sessão apresentou diferenças significativas em relação à todas as sessões e a $2^{a}$ sessão apresentou diferenças significativas em relação às sessōes 6 e 7. Já no GAS, foram detectadas diferenças significativas entre as sessōes de prática $(\mathrm{F}[7 ; 77]=6,61 ; \mathrm{p}=0,0001)$. O teste "Post Hoc" mostrou que a $1^{\text {a }}$ sessão apresentou diferenças significativas em relação à todas as sessōes. Finalmente, no GBS, foram detectadas também diferenças significativas entre as sessôes de prática $(\mathrm{F}[7 ; 91]=3,59 ; \mathrm{p}=0,02)$. O teste "Post Hoc" localizou diferenças significativas em apenas duas sessōes, a $1^{\text {a }}$ e $7^{\text {a }}$ sessão. Com base nesses resultados foi verificada a melhora de desempenho em todos os grupos ao longo da fase de aquisição.

\section{Fase de transferência}

Nos participantes com nível inferior de habilidade foram encontradas diferenças significativas no fator testes $(\mathrm{F}[2 ; 56]=10,09 ; \mathrm{p}=0,0001)$. O teste "Post Hoc" indicou que na $8^{\text {a }}$ sessão o desempenho foi significativamente superior em relação ao do teste de transferência imediato, contudo entre o teste de transferência imediato e o retardado não foram evidenciadas diferenças significativas. Não foram detectadas diferenças significativas no fator tipo de prática $(\mathrm{F}[1 ; 28]=1,72 ; \mathrm{p}=0,058)$ e nem na interação tipo de prática e testes $(\mathrm{F}[1 ; 28]=1,72 ; \mathrm{p}=0,058)$.

Em participantes com nível superior de habilidade, foram encontradas diferenças significativas no fator testes $(\mathrm{F}[2 ; 48]=9,45 ; \mathrm{p}=0,0001)$. $\mathrm{O}$ teste "Post Hoc" indicou que na $8^{\text {a }}$ sessão o desempenho foi significativamente superior em relação ao dos testes de transferência imediato e o retardado, contudo, os dois testes de transferência não foram estatisticamente diferentes entre si.

Foram detectadas diferenças significativas ( $\mathrm{F}[1$; $24]=10,13 ; \mathrm{p}=0,004)$ no fator tipo de prática e o teste "Post Hoc" indicou superioridade do GBS em relação ao GBS no teste de transferência imediato. Contudo não houve diferenças estatísticas entre ambos os grupos no teste de transferência retardado.

Finalmente, foram constatadas diferenças significativas também no fator interação tipo de prática e testes $(\mathrm{F}[2 ; 48]=5,95 ; \mathrm{p}=0,005)$. O teste "Post Hoc" indicou que o GAS teve desempenhos diferentes nos dois testes de transferência, sendo melhor no imediato e pior no retardado, e apresentou diferenças significativas também entre a $8^{a}$ sessão da aquisição 
e o teste de transferência retardado. Já o grupo de prática em blocos apresenta diferenças significativas entre a $8^{a}$ sessão da aquisição e o teste de transferência imediato, não havendo diferenças entre os dois testes de transferência. Esses resultados confirmam que as participantes com nível de habilidade superior têm melhores resultados no teste de transferência imediato quando são submetidas à prática aleatória.

\section{Discussão}

O presente estudo investigou o EIC tendo em conta o nível de habilidade das participantes. Quanto ao tipo de prática com participantes com nível de habilidade superior, o GAS foi superior da primeira à última sessão em relação ao GBS. Neste caso, a superioridade do GAS pode estar relacionada aos elevados níveis de processamento e de atenção requisitados na práctica aleatória. No caso das participantes com nível de habilidade inferior, os melhores resultados do GBI podem estar ligados ao processo de obtenção da ideia do movimento facilitado pela prática em blocos (Gentile, 1972).

Nos testes de transferência, em sujeitos com nível de habilidade superior, destaca-se o fato de o GAS apresentar diferenças significativas no teste imediato. Em relação à hipótese de que haveria diferenças significativas entre os grupos de prática nos testes de transferência imediato e retardado, estes resultados confirmam parcialmente a mesma, visto que nenhuma diferença foi constatada no teste de transferência retardado. Pode-se argumentar que as fontes de informação a que foram submetidos as participantes da prática aleatória podem ter favorecido o GAS no teste de transferência imediato. Segundo LeE e MagiLl (1985) os sujeitos submetidos à prática aleatória têm experiência com os parâmetros, o conhecimento de resultados, as consequências sensoriais e as condiçôes iniciais com maior amplitude, comparados aos sujeitos que praticam em blocos.

A superioridade do GAS, apenas no teste imediato também foi verificada nos estudos de DeL Rey et al. (1994) e SMith (1997). De acordo com muitos autores, a dissipação da vantagem da prática aleatória pode ser um forte indício de que o EIC é temporário (Del Rey et al., 1994; Meira Júnior, 1999; Newell \& Russell, 2007; Smith, 1997). Por outro lado, SMith (1997) afirma que a atenção elevada causada pela prática aleatória previne o decréscimo do desempenho nas primeiras tentativas do teste de transferência imediato; porém, com o decorrer das sessões, os sujeitos da prática em blocos recuperam a desvantagem inicial. Importa lembrar que, no presente estudo, as participantes realizaram o teste de transferência imediato no último dia da fase de aquisição, e entende-se que os efeitos da elevada ativação e consequente maior demanda de atenção para as tarefas provocadas pela prática aleatória, ainda perduravam. De acordo com LeE, Wulf e SCHMidt (1992) e MEIRA Júnior (1999), as vantagens da prática aleatória na aquisição de tarefas motoras complexas estão relacionadas aos aspectos temporários da prática, não necessariamente a fatores de aprendizagem efetiva, caracterizada por efeitos permanentes. Portanto, o EIC tem muitas hipóteses de dissipação ao longo das tentativas prolongadas dos testes, o que o caracterizaria como um fator de efeito transitório (LeE, Wulf \& Schmidt, 1992; Meira Júnior, 1999; Newell \& Russell, 2007).

Neste estudo, constatou-se um parco desempenho do GBS nos testes, embora este tenha obtido um desempenho significativo na fase de aquisição em relação aos grupos com nível de habilidade inferior. Isso vem a confirmar o argumento de que o EIC ocorre em indivíduos habilidosos submetidos à prática aleatória (Del Rey, 1989; Hall, Domingues \& Cavazos, 1994). Contudo, o mesmo fator põe em desvantagem os sujeitos com nível de habilidade inferior, por estes serem insensíveis aos benefícios da elevada interferência contextual, aliado a sua limitada capacidade de relacionar diversas fontes de informação simultaneamente. As participantes com nível de habilidade inferior (GBI e GAI) registaram baixas prestações em todos os testes de transferência. Esses resultados corroboram os demais estudos com crianças e novatos - indivíduos com baixos níveis de habilidade. Estes sujeitos demoram mais para decifrar as diferenças e similaridades entre habilidades relacionadas, havendo necessidade de serem submetidos a grande quantidade de prática em blocos antes da prática aleatória (BorToli, RobazZA, DURIGON \& CARRA, 1992; Corrêa \& Pellegrini, 1996; Del Rey, WuGHALTER, WhiteHURST \& BARNWELL, 1983; EDWARDS, Elliott \& Lee, 1986; FARrow \& MASChetTe, 1997; French, Rink \& Werner, 1990; Freudenheim \& Tani, 1995; Santos, 1997; Ugrinowitsch, 1997; Ugrinowitsch \& MaNoel, 1996; Wegman, 1999). 
Esse resultado corrobora os estudos em que indivíduos menos habilidosos tendem a não confirmar o EIC (Hebert, Landin \& Solmon, 1996), sendo os fatores que podem estar ligados ao fracasso destes, a quantidade de prática e a dificuldade das tarefas de campo. O efeito das várias condiçôes de prática na aprendizagem motora, considerada a relação entre a complexidade da tarefa e o nível de habilidade dos aprendizes continua a ser um grande desafio aos estudos da área (GuADAGNOLI \& LEE, 2004).

Partindo do pressuposto do EIC, segundo o qual o aumento da variabilidade ao longo das tentativas pode produzir um processamento mais distinto e mais elaborado, o que leva a um baixo desempenho na aquisição e uma melhoria nos testes de transferência e retenção (BATTIG, 1972), pode-se afirmar que em estudos de campo isso não é tão claro. A hipótese de ocorrência do EIC é mais válida para as pesquisas de laboratório (MEIRA JúNIOR, 1999). Fatores que podem interferir nessa tendência podem ser o nível de dificuldade das tarefas e a quantidade de prática. Em tarefas de laboratório o EIC se observa porque as tarefas são mais simples e, portanto, demandam menos prática. Em contrapartida, na prática de habilidades desportivas, por exemplo, é possível que o EIC não ocorra, pelo menos até que os sujeitos sejam submetidos a um grande número de repetições. Em outras palavras, a quantidade de prática é um dos fatores que podem influenciar os resultados, na medida em que as tarefas de campo são muito complexas, exigindo um grande número de tentativas para que sejam adquiridas com elevados graus de proficiência (HEBERT, LANDIN \& Solmon, 1996; Shea, Kohl \& Indermill, 1990). Pode ser que se o número de tentativas de aquisição fosse maior (mais de 24 por sessão) no presente estudo, o EIC pudesse ser observado.

As características dos serviços manipulados no presente estudo, apesar de apresentarem algumas similaridades, possuem diferenças marcantes que podem ter dificultado a ocorrência do EIC. As similaridades foram: a sequência do movimento (posição, lançamento da bola, ataque à bola e finalização), a batida na bola, a sincronização da soltura da bola com o movimento do braço, e a transferência do peso do corpo da perna de trás para a perna da frente no momento do ataque à bola. Por outro lado, as diferenças entre os serviços foram: os padróes de movimento utilizados, a posição em relação ao alvo, o "timing", a altura de ataque à bola e a utilização de diferentes grupos musculares. Assim, as diferenças entre os serviços constituíram fatores para a ausência do EIC em sujeitos com nível de habilidade inferior, partindo-se do princípio de que estes demoram a decifrar as diferenças e similaridades entre as habilidades.

Em conformidade com a literatura, como já se referiu anteriormente, o EIC parece não ser generalizado porque ocorre em situações específicas. De acordo com vários autores, a prática aleatória supera a prática em blocos geralmente: a) em situações de laboratório; b) com sujeitos habilidosos; c) mais na transferência que na retenção; e d) com prática extensiva (BRADY, 2004; GiUfFrida, SHeA \& Fairbrother, 2002; Meira JúNIOR, TANi \& Manoel, 2001; Meira Júnior \& TANi, 2005). Contudo, conforme os mesmos autores, sujeitos submetidos à prática em blocos também superam os submetidos à prática aleatória, quando estes são crianças ou adolescentes com pouca quantidade de prática.

Considerando as características do presente estudo - de campo, com tarefas complexas do mundo real - os resultados obtidos permitem concluir que a prática aleatória é superior à prática em blocos em sujeitos habilidosos, visto que participantes com nível de habilidade superior mostraram melhores resultados no teste de transferência imediato quando praticam de forma aleatória. No entanto, a ausência de diferenças significantes entre os grupos com nível de habilidade superior no teste de transferência retardado sugere que o EIC pode ter sido temporário (Lee, Wulf \& Schmidt, 1992; Meira Júnior, 1999; Newell \& Russell, 2007).

Um tema para futuras investigaçóes, sobretudo para sujeitos com nível de habilidade superior, é quando introduzir a variação na prática. Alguns pesquisadores têm proposto práticas mistas (CoRrêa, 2001; Corrêa, Barros, Massigli, Gonçalves \& Tani, 2007; Paroli, 2005), uma tendência que aponta para a mesma direção proposta nos modelos descritivos dos estágios de aprendizagem motora: prática com pouca variação no início e mais variação no final (Tani, Meira Júnior \& Cattuzzo, 2010). Em outras palavras, para garantir ganhos de aprendizagem em sujeitos com nível de habilidade superior, a adoção de prática em blocos somente nos momentos iniciais do processo, antes que qualquer variabilidade seja introduzida, pode ser mais vantajosa para a compreensão do padrão básico do movimento (CORRÊA et al., 2007; PAroli, 2005; Wrisberg, 2007). Depois de adquirir o padrão do movimento, poder-se-ia iniciar a sua variação a cada tentativa. Em caso de aprendizes menos habilidosos, uma prática extensiva (muitas tentativas) com prática constante, seguida da prática em blocos poderia ser mais eficaz. São temas que ficam para estudos futuros na busca de evidências empíricas mais consistentes acerca do EIC. 


\begin{abstract}
Contextual interference and level of skill in the learning of volleyball serve
\end{abstract}

The purpose of this study was to investigate the effect of contextual interference on acquiring the volleyball serve in adolescents with different skill levels, considering then immediate and delayed transfer tests. Participants were 56 schoolgirls separated in four practice groups: random practice with higher level skill subjects (GAS), blocked practice with higher level skill subjects (GBS), random practice with lower level skill subjects (GAI) and blocked practice with lower level skill subjects (GBI). The acquisition task consisted in performing underhand and overhead services directed at two targets affixed to the ground. The results showed no effect of contextual interference on the lower level skill subjects. However when comparing the types of practice between the higher level skill subjects, the effect was observed in the immediate transfer test. The absence of significant differences between groups in the delayed transfer test suggest that the contextual interference effect may be temporary.

UnITERMS: Variable practice; Motor learning; Motor skill level.

\title{
Resumen
}

Interferencia contextual y nivel de habilidad en el aprendizaje del servicio de voleibol

Este estudio investigó el efecto de la interferencia contextual (IC) en la adquisición del servicio de voleibol en adolescentes con diferentes niveles de habilidad, teniendo en cuenta las pruebas de transferencia inmediata y retardada. La muestra estuvo conformada por 56 niñas de edades comprendidas entre 14 y 16 años, divididas en cuatro grupos de práctica: al azar con mayor nivel de habilidad (GAS), por bloques con mayor nivel de habilidad (GBS), al azar con menor nivel de habilidad (GAI ) y por bloques con menor nivel de habilidad (GBI). La tarea motora consistió de servicios por abajo y por encima dirigidos a objetivos en la cancha. Los resultados mostraron que no se observó el efecto de la IC en los grupos GAl y $\mathrm{GBI}$, por otra parte, los grupos GAS y GBS presentaron mejor rendimiento en la prueba de transferencia inmediata. La ausencia de diferencias significativas en la prueba de transferencia de retraso sugiere que el efecto de la IC puede ser temporario.

Palabras clave: Práctica varió; Aprendizaje motor; Nivel de habilidad.

\section{Referências}

AMERICAN ASSOCIATION FOR HEALTH, PHYSICAL EDUCATION, RECREATION AND DANCE (AAHPERD). Skills test manual: volleyball for boys and girls. Washington: AAHPERD, 1969.

BATTIG, W.F. Facilitation and interference. In: BILODEAU, E.A. (Ed.). Acquisition of skill. New York: Academic Press, 1996. p.215-44.

Intra task interference as a source of facilitation in transfer and retention. In: THOMPSON, R.F.; VOSS, J.F. (Eds.). Topics in learning and performance. New York: Academic Press, 1972. p.131-59.

BRADY, F. Contextual interference and teaching golf skills. Perceptual and Motor Skills, Missoula, v.84, p.334-50, 1997. . A theoretical and empirical review of the contextual interference effect and the learning of motor skills. Quest, Champaign, v.50, p.266-93, 1998.

Contextual interference: a meta-analytic study. Perceptual and Motor Skills, Missoula, v.99, p.116-26, 2004.

BORTOLI, L.; ROBAZZA, C.; DURIGON, V.; CARRA, C. Effects of contextual interference on learning technical sport skills. Perceptual and Motor Skills, Missoula, v.75, p.555-62, 1992. 
CORRÊA, U.C. Estrutura da prática e processo adaptativo na aquisição de habilidades motoras. 2001. Tese (Doutorado) - Escola de Educação Física e Esporte, Universidade de São Paulo, São Paulo, 2001.

CORRÊA, U.C.; BARROS, J.A.C.; MASSIGLI, M.; GONÇALVES, L.A.; TANI, G. A prática constante-aleatória e o processo adaptativo de aprendizagem motora: efeitos da quantidade de prática constante. Revista Brasileira de Educação Física e Esporte, São Paulo, v.21, p.301-14, 2007.

CORRÊA, U.C.; PELLEGRINI, A.M. A interferência contextual em função do número de variáveis. Revista Paulista de Educação Física, São Paulo, v.10, p. 21-33, 1996.

DEL REY, P. Training and contextual interference effects on memory and transfer. Research Quarterly for Exercise and Sport, Washington, v.60, p.342-47, 1989.

DEL REY, P.; WUGHALTER, E.H.; WHITEHURST, M.; BARNWELL, J. Contextual interference and experience in acquisition and transfer. Perceptual and Motor Skills, Missoula, v.57, p.241-42, 1983.

DEL REY, P.; XIAOYING, L.; SIMPSON, K.J. Does retroactive inhibition influence contextual interference effects? Research Quarterly for Exercise and Sport, Washington, v.65, p.120-26, 1994.

EDWARDS, J.M.; ELLIOTT, D.; LEE, T.D. Contextual interference effects during skill acquisition and transfer in Down's syndrome adolescents. Adapted Physical Activity Quarterly, Champaign, v.3, p.250-58, 1986.

FARROW, D.; MASCHETTE, W. The effects of contextual interference on children learning forehand tennis groundstroke. Journal of Human Movement Studies, London, v.33, p.47-67, 1997.

FRENCH, K.E.; RINK, J.E.; WERNER, P.H. Effects of contextual interference on retention of three volleyball skills. Perceptual and Motor Skills, Missoula, v.71, p.179-86, 1990.

FREUDENHEIM, A.M.; TANI, G. Efeitos da estrutura de prática variada na aprendizagem de uma tarefa de timing coincidente em crianças. Revista Paulista de Educação Física, São Paulo, v.9, n.2, p.87-98, 1995.

GENTILE, A.M. A working model of skill acquisition with application to teaching. Quest, Champaign, v.17, p.3-23, 1972. GIUFRIDA, C.G.; SHEA, J.B.; FAIRBROTHER, J.T. Differential transfer benefits of increased practice for constant, blocked, and serial practices schedules. Journal of Motor Behavior, Washington, v.34, n.4, p.353-65, 2002.

GOODWIN, J.E.; MEEUWSEN, H.J. Investigation of the contextual interference effect in the manipulation of the motor parameter of over-all force. Perceptual and Motor Skills, Missoula, v.83, p.735-43, 1996.

GUADAGNOLI, M.A.; HOLCOMB, W.R.; WEBER, T.J. The relationship between contextual interference effects and performer expertise on the learning of a putting task. Journal of Human Movement Studies, London, v.37, p.19-36, 1999. GUADAGNOLI, M.A.; LEE, T.D. Challenge point: a framework for conceptualizing the effects of various practice conditions in motor learning. Journal of Motor Behaviour, Washington, v.36, p.212-24, 2004.

HALL, K.G.; DOMINGUES, D.A.; CAVAZOS, R. Contextual interference effects with skilled baseball players. Perceptual and Motor Skills, Missoula, v.78, p.835-41, 1994.

HEBERT, E.P.; LANDIN, D.; SOLMON, M.A. Practice schedule effects on the performance and learning of low- and high-skilled students: an applied study. Research Quarterly for Exercise and Sport, Washington, v.67, p.52-58, 1996.

JONES, L.L.; FRENCH, K.E. Effects of contextual interference on acquisition and retention of three volleyball skills. Perceptual and Motor Skills, Missoula, v.105, n.3, p.883-90, 2007.

LANDIN, D.; HERBERT, E.P. A comparison of three practice schedules along the contextual interference continuum. Research Quarterly for Exercise and Sport, Washington, v.68, p.357-61, 1997.

LEE, T.D.; WULF, G.; SCHMIDT, R.A. Contextual interference in motor learning dissociates effects due to the nature of task variations. The Quarterly Journal of Experimental Psychology, London, v.44A, p.627-44, 1992.

LEE, T.D.; MAGILL, R.A. Can forgetting facilitate skill acquisition? In: GOODMAN, D.; WILBERG, R.B.; FRANKS, I.M. (Eds.). Differing perspectives in motor learning, memory, and control. Amsterdam: North-Holland, 1985. p.3-22. MEIRA JÚNIOR, C.M. O efeito da interferência contextual na aquisiçáo da habilidade saque do voleibol em crianças: temporário, duradouro ou inexistente? 1999. Dissertação (Mestrado) - Escola de Educação Física e Esporte, Universidade de São Paulo, São Paulo, 1999. MEIRA JÚNIOR, C.M.; TANI, G. Interferência contextual em aprendizagem motora: o paradoxo é uma realidade? In: TANI, G. (Ed.). Comportamento motor: aprendizagem e desenvolvimento. Rio de Janeiro: Guanabara Koogan, 2005. p.223-34. . The contextual interference effect in acquisition of dark- throwing skill tested on a transfer test with extended trials. Perceptual and Motor Skills, Missoula, v.92, p.910-18, 2001.

MEIRA JÚNIOR, C.M.; TANI, G.; MANOEL, E. A estrutura de práctica variada em situações de ensino e aprendizagem. Revista Brasileira de Ciência e Movimento, São Caetano do Sul, v.9, p.55-64, 2001.

NEWELL, K.M.; RUSSELL, D.M. How persistent and general is the contextual interference effect? Research Quarterly for Exercise and Sport, Washington, v.78, n.4, p.318-27, 2007. 
PAROLI, R. Efeito da estrutura de prática na aquisição de uma habilidade motora. 2005. Dissertação (Mestrado) Escola de Educação Física e Esporte, Universidade de São Paulo, São Paulo, 2005.

POLLOCK, B.J.; LEE, T.D. Dissociated contextual interference effects in children and adults. Perceptual and Motor Skills, Missoula, v.84, p.851-58, 1997.

SANTOS, L.H.R. Interferência contextual: organização das prácticas em blocos e randômica na aprendizagem do futebol. 1997. Dissertação (Mestrado) - Universidade Federal de Santa Maria, Santa Maria, 1997.

SCHMIDT, R.A. A scheme theory of discrete motor skill learning. Psychological Review, Washington, v.82, p.225-260, 1975. SMITH, P.J.K. Attention and the contextual interference effect for a continuous task. Perceptual and Motor Skills, Missoula, v.84, p.82-92, 1997.

SHEA, C.H.; KOHL, R.; INDERMILL, C. Contextual interference: contributions of practice. Acta Psychologica, Amsterdam, v.73, p.145-57, 1990.

SHEA, J.B.; MORGAN, R.L. Contextual interference effects on the acquisition, retention and transfer of a motor skill. Journal of Experimental Psychology: Human Learning and Memory, Washington, v.5, n.2, p.179-87, 1979.

TANI, G. Aprendizagem motora e esporte de rendimento: um caso de divórcio sem casamento. In: BARBANTI, V.; AMADIO, A.; BENTO, J.; MARQUES, A. (Orgs.). Esporte e atividade física. São Paulo: Manole, 2002. p.145-62.

Equivalência motora, variabilidade e graus de liberdade: desafios para o ensino dos jogos desportivos. In: TAVARES, F.; GARGANTA, J.; GRAÇA, A.; MESQUITA, I. (Eds.). Olhares e contextos de performance nos jogos desportivos. Porto: Universidade do Porto Editorial, 2008. p.85-92.

TANI, G.; MEIRA JÚNIOR, C.M.; CATTUZZO, M.T. Aprendizagem motora e educação física: pesquisa e intervenção. In: BENTO, J.O.; TANI, G.; PRISTA, A. (Orgs.). Desporto e educação física em português. Porto: Multitema, 2010. p.36-56. TEMPRADO, J.J. Apprentissage moteur: quelques données actuelles. Education Physique et Sport, Paris, v.267, p.20-23, 1997. TRAVLOS, A.K. Specific and variability of practice, and contextual interference in acquisition and transfer of an underhand volleyball serve. Perceptual and Motor Skills, Missoula, v.110, n.1, p.298-312, 2010.

UGRINOWITSCH, H. Interferência contextual: manipulação de programas e parâmetros na aquisição da habilidade saque do voleibol. 1997. Dissertação (Mestrado) - Escola de Educação Física e Esporte, Universidade de São Paulo, São Paulo, 1997. WRISBERG, C.A. Sport skills instruction for coaches. Champaign: Human Kinetics, 2007.

ZETOU, E.; MICHALOPOULOU, M.; GIAZITZI, K.; KIOUMOURTZOGLOU, E. Contextual interference effects in learning volleyball skills. Perceptual and Motor Skills, Missoula, v.104, n.3, p.995-1004, 2007.

\begin{tabular}{r|l} 
ENDEREÇo & \\
Go Tani & \\
Escola de Educação Física e Esporte - USP & Recebido para publicação: 01/09/2011 \\
Av. Prof. Mello Moraes, 65 & Revisado: 09/05/2012 \\
05508-030 - São Paulo - SP - BRASIL & Aceito: 25/05/2012 \\
e-mail: gotani@usp.br & \\
&
\end{tabular}

740 • Rev. bras. Educ. Fís. Esporte, São Paulo, v.26, n.4, p.731-40, out./dez. 2012 